\title{
Sociedad ERAS y Latinoamérica
}

\author{
ERAS Society and Latin America \\ Sociedade ERAS e América Latina
}

\author{
Santiago Mc Loughlin ${ }^{1 *}$, Adrian O. Alvarez ${ }^{1}$, Olle Ljungqvist ${ }^{2}$ \\ Recibido: 1 de junio de 2019. Aceptado para publicación: 5 de agosto de 2019. \\ Publicado en línea: 9 de noviembre de 2019 \\ https://doi.org/10.35454/rncm.v3n1.020
}

\section{Resumen}

El volumen de cirugías y su complejidad aumenta de forma constante en el mundo. En nuestra región, esta demanda no resuelta, ni en cantidad ni en calidad de atención, además de la deficiencia en el registro del proceso de cuidado conduce a grandes desperdicios de dinero, tiempo y energía de los sistemas de salud y sus profesionales.

Los objetivos de ERAS $^{\circledR}$ (Enhanced Recovery After Surgery) incluyen desarrollar el cuidado perioperatorio y mejorar su recuperación a través de la investigación, educación, auditoría e implementación de prácticas basadas en la evidencia. Registrar sistemáticamente el proceso de cuidado, actuar con base en el diagnóstico $y$, por último, auditar y ajustar las acciones constituyen los principios de trabajo común a todos los protocolos ERAS $^{\circledast}$. Las diferentes etapas de la atención quirúrgica son abordadas como un proceso indivisible que constituye la atención perioperatoria a través de un equipo multidisciplinario. Este equipo trabaja semana a semana utilizando el sistema de gestión de datos de ERAS Society que provee un registro con variables estandarizadas mundialmente lo que permite la auditoría interna y la comparación externa.

La implementación de programas ERAS $^{\circledR}$ demostró, en el ámbito mundial, reducciones significativas en la estancia hospitalaria, complicaciones postoperatorias y los costos de atención. En el ámbito regional los protocolos ERAS ${ }^{\circledR}$ se han expandido estando al momento

\section{Summary}

The volume of surgeries and their complexity has been increasing steadily worldwide. In our region, this demand is not resolved in quantity or quality and the lack of register of the care process leads to great misspending of funds, time and energy from health systems and their professionals.

The goal of ERAS ${ }^{\circledR}$ (Enhanced Recovery After Surgery) is to develop perioperative care and improve its recovery through research, education, auditing and implementation of evidence-based practices. Systematically registration of the care process, actions based on accurate diagnosis and, finally, audit and adjustments of the actions are the working principles common to all ERAS ${ }^{\circledR}$ protocols. The different stages of surgical care are approached by a multidisciplinary team as an indivisible process that constitutes perioperative care. This team works week by week using the ERAS Society data management system that provides a registry with variables globally standardized that allow internal audit and external comparison.

The implementation of ERAS ${ }^{\circledR}$ programs in Europe and North America showed significant reductions in hospital stay, postoperative complications and costs of care. At a regional level, the ERAS $^{\circledR}$ protocols have expanded to 6 countries and 10 centers with results similar to those reported in the rest of the world. A major change in perioperative care is underway in the region, and our goal is to make it available to everyone.

\section{Resumo}

O volume de cirurgias e sua complexidade aumenta constantemente no mundo. Na nossa região, essa procura não atendida, nem em quantidade nem em qualidade de assistência, além de uma deficiência no registro do processo de assistência, gera grande desperdício de dinheiro, tempo e energia nos sistemas de saúde e nos seus profissionais.

Os objetivos do ERAS $^{\circledR}$ (Enhanced Recovery After Surgery) incluem o desenvolvimento de cuidados perioperatórios e a melhoria da recuperação por meio de pesquisa, educação, auditoria e implementação de práticas baseadas em evidências. Registar sistematicamente o processo de cuidar, atuar com base no diagnóstico e por fim auditar e ajustar os procedimentos, constituem os princípios de trabalho comuns a todos os protocolos ERAS ${ }^{\oplus}$. As diferentes etapas do atendimento cirúrgico são abordadas como um processo indivisível que constitui o cuidado perioperatório por meio de uma equipe multidisciplinar. Essa equipe trabalha semana após semana usando o sistema de gestão de dados da Sociedade ERAS, que fornece um registo com variáveis estandardizadas mundialmente, o que permite uma auditoria interna e a comparação externa.

A implementação dos programas ERAS $^{\circledR}$ demonstrou, em todo o mundo, reduções significativas no tempo de internamento hospitalar, nas complicações pós-operatórias e nos custos com os cuidados. A nível regional, os protocolos ERAS $^{\circledR}$ se expandiram estando neste mo- 
presentes en 6 países y 10 centros de atención con resultados similares a los reportados en el mundo. Un gran cambio en el cuidado perioperatorio está en marcha en la región, y nuestro objetivo, es hacer que esté disponible para todos.

Palabras clave: Enhanced Recovery After Surgery, cuidado perioperatorio.
Keywords: Enhanced Recovery After Surgery; Perioperative care. mento presentes em 6 países e 10 centros de atendimento com resultados semelhantes aos relatados pelo mundo. Uma grande mudança no atendimento perioperatório está em andamento na região, e o nosso objetivo é que esteja disponível para todos.

Palavras-chave: Enhanced Recovery After Surgery, cuidados perioperatórios.
Servicio de Anestesiología, Hospital Italiano de Buenos Aires, Buenos Aires, Argentina.

*Correspondencia: Santiago Mc Loughlin santiago.mcloughlin@hospitalitaliano.org.ar

\section{INTRODUCCIÓN}

El volumen de cirugías y su complejidad ha presentado un aumento constante en las últimas décadas en el mundo ${ }^{(1,2)}$. En nuestra región, esta demanda no se encuentra resuelta en cantidad ni en calidad de atención (no se realizan todas las cirugías necesarias y los resultados son pobremente auditados) ${ }^{(3)}$. Este desequilibrio da como resultado un número inaceptable de muertes y complicaciones prevenibles ${ }^{(4)}$. Un problema agregado es el registro muy deficitario de las mismas, con lo cual es difícil estimar la gravedad de la crisis perioperatoria en Latinoamérica. Desafortunadamente, no parecen existir prioridades políticas para el desarrollo del cuidado perioperatorio en el futuro cercano de nuestra región. Como resultado, los profesionales de salud involucrados en los equipos quirúrgicos debemos enfrentar este desafío con nuestras propias herramientas.

\section{LA SOCIEDAD ERAS}

La sociedad para la recuperación acelerada luego de la cirugía (Enhanced Recovery After Surgery, ERAS ${ }^{\varpi}$ ) surge en el año 2001 en Suecia como una iniciativa de varios grupos quirúrgicos interesados en promover un cuidado multimodal e integral del paciente quirúrgico $^{(5,6)}$. El objetivo de ERAS ${ }^{\circledR}$ es desarrollar el cuidado perioperatorio y mejorar su recuperación a través de la investigación, educación, auditoría e implementación de prácticas basadas en la evidencia ${ }^{(6)}$. Si bien el contenido de las guías clínicas de ERAS $^{\circledR}$ puede variar dependiendo de la práctica quirúrgica abordada, se repite siempre una secuencia lógica de acción común: analizar los datos, planear y actuar con base en el diagnóstico de situación, auditar los resultados de la acción y, por último, ajustar las conductas basados en la auditoría. Este ciclo de mejoría continua es la base del sistema de cuidados ERAS y la modalidad de trabajo en equipo semana a semana.

\section{IMPLEMENTACIÓN DEL PROTOCOLO ERAS Y LA PLATAFORMA EN LÍNEA EIAS}

Contar con datos confiables es indispensable para empezar a trabajar. Sin embargo, en nuestra región, la información recolectada por los gobiernos o grandes instituciones a menudo es ineficiente. El resultado final es la incapacidad de hacer un diagnóstico real y auditar resultados y procesos, situación que conduce a grandes desperdicios de dinero, tiempo y energía de los sistemas de salud y sus profesionales.

La implementación del programa ERAS $^{\circledR}$ permite que la recopilación de los datos del cuidado perioperatorio ya no dependa de instituciones centralizadas ${ }^{(7)}$. La información confiable está ahora a nuestro alcance a través de la plataforma en línea EIAS (ERAS Interactive Audit System) de la sociedad ERAS ${ }^{\circledR}$. La recolección electrónica de datos utilizando EIAS evita problemas comunes, como la falta de registros médicos claros y provee un almacenamiento cuyas variables se encuentran estandarizadas para todos los centros en el mundo. Esto permite realizar con facilidad comparaciones entre diferentes períodos de una misma entidad de salud o entre diferentes instituciones. Es importante destacar 
que este sistema de gestión de datos, auditoría interna y comparación externa requiere solo acceso a la Internet. Incluso con el esfuerzo aislado de un profesional comprometido con el registro de sus pacientes en la base de datos (EIAS) ya es posible tener un diagnóstico de su situación.

\section{EL ENFOQUE MULTIDISCIPLINARIO}

Dado que la atención quirúrgica es un esfuerzo conjunto de especialistas de varias áreas (anestesiólogos, cirujanos, enfermeros, nutricionistas, epidemiólogos, especialistas en tecnologías de la información, ingenieros, agentes de comunicación, educadores, estadísticos, etc.) la formación de un equipo multidisciplinario resulta indispensable. En los programas ERAS ${ }^{\varpi}$, las diferentes etapas de la atención quirúrgica no se tratan de forma individual, sino como un proceso indivisible que constituye la atención perioperatoria. La información adecuada permite identificar las deficiencias que deben abordarse y corregirse; pero además de ello, es necesaria una comunicación efectiva entre los miembros del sistema de atención médica para comprender la naturaleza real de los problemas y planear sus posibles soluciones. Independientemente del tipo de procedimiento quirúrgico abordado, todos los programas de implementación ERAS comienzan con la formación de un equipo de trabajo que tiene como objetivo fijar reuniones semanales donde se analizarán diferentes situaciones y se planificarán las acciones correspondientes. Estas reuniones semanales son clave para reforzar los canales de comunicación efectiva y poder asegurar el cambio.

\section{LAS GUÍAS DE MANEJO Y EL SISTEMA DE AUDITORÍA CONTINUA}

Como hoja de ruta para guiar nuestro trabajo asistencial diario, ERAS ${ }^{\circledast}$ Society ha desarrollado guías de manejo clínico con contenido específico según el tipo de procedimiento. El acceso a estas guías es irrestricto a través del sitio web de la sociedad (www.erassociety. org) y provee información basada en la evidencia para estandarizar nuestras prácticas. Sin embargo, a pesar de ser un tema académico candente y el éxito alcanzado en otras industrias, la estandarización en la atención de la salud ha encontrado dificultades para ser adoptada. Con respecto a este asunto, Janet Woodcock, directora del Centro de Evaluación e Investigación de Medicamentos de la FDA, identificó la división entre la investigación y la práctica clínica de la medicina como uno de los problemas más críticos que se enfrentan hoy. La baja tasa de adherencia a las guías de manejo se refleja en un estudio que indicó que los pacientes reciben en promedio solo $54,9 \%$ de las pautas que los distintos centros médicos refieren utilizar en forma cotidiana ${ }^{(8)}$. Es en este aspecto que la aplicación de las guías ERAS difiere sustancialmente de otras guías de manejo clínico existentes. Utilizando los datos registrados en el EIAS, la adherencia a las recomendaciones clínicas que el equipo perioperatorio dice seguir es auditada de forma constante por ellos mismos y su implementación real reforzada semana a semana en las reuniones de equipo. Esta auditoría permite ajustar el sistema de cuidado a partir de la rápida identificación de deficiencias o el refuerzo de intervenciones exitosas. Otro aspecto interesante del sistema de auditoría continua es que la evidencia para futuras guías de manejo puede surgir o ser modificada desde la práctica diaria y no, como es habitual, surgir únicamente desde un escenario diseñado para la investigación clínica. Esta característica no solo ayuda en el desarrollo de un sistema de salud de autoaprendizaje, sino que también brinda una gran oportunidad para desarrollar investigaciones en regiones, que en general, solo reciben financiamiento adecuado cuando participan en un ensayo clínico aleatorizado patrocinado por la industria.

\section{ERAS EN AMÉRICA LATINA (ERAS LATAM)}

Sobre la base de todo lo anterior, ERAS constituye una opción lógica para abordar nuestros problemas complejos y los programas respaldados por la $E R A S^{\circledast}$ Society proporcionan la estructura de gestión que, de lo contrario, estaria ausente en nuestro contexto.

Afortunadamente, en varios países de América Latina esto ya no es solo un sueño o un proyecto. Los protocolos $\mathrm{ERAS}^{\circledast}$ se expandieron y continúan haciéndolo en distintas especialidades y hospitales de nuestro continente. El punto de partida de ERAS en Latinoamérica fue el programa de implementación liderado por Robin Kennedy, Olle Ljungqvist y Jennifer Burch para el «Hospital Italiano de Buenos Aires» de Argentina. Esto resultó en el primer centro de excelencia de la región en 2014. En el año 2015, se sumaron a esta iniciativa un equipo de Colombia (Clínica Reina Sofía Org. Sanitas) y uno de México (Hospital Civil de Guadalajara) siendo ambos, hoy en día, centros de excelencia acreditados por la sociedad ERAS. En 2016, dos instituciones uruguayas (CAMOC de Carmelo y la Médica Uruguaya Corporación de Asistencia Médica, de Montevideo) también se unieron a nues- 
tros esfuerzos para mejorar la atención perioperatoria en la región y en la actualidad se encuentran acreditados como centros de excelencia. Del mismo modo, en 2016, dos grandes centros médicos brasileños y referentes regionales iniciaron sus programas de implementación en Brasil (Hospital Israelita Albert Einstein de San Pablo y Santa Casa de la Misericordia de Porto Alegre). Finalmente, y mostrando que el avance de ERAS en la región sigue vigente, en el último año la Clínica Alemana de Santiago, el Sanatorio Güemes de Argentina y el Instituto Nacional de Cancerología de México, también se sumaron a la iniciativa.

Gracias a estos esfuerzos, la implementación de los programas ERAS $^{\circledast}$ en la región ha comenzado a mostrar resultados alentadores similares a los reportados en el resto del mundo ${ }^{(9,10)}$. El análisis de 1.216 pacientes sometidos a cirugía colorrectal en 10 centros de la región con programas ERAS en curso, encontró que la mediana del cumplimiento de estos protocolos en la región aumentó de $36 \%$ en el primer trimestre de 2015 a $64 \%$ en el último trimestre de 2018. Así mismo, el cumplimiento de los protocolos de ERAS demostró un impacto significativo en la duración de la estancia hospitalaria (Figura 1). El aumento del cumplimiento también se asoció a una menor internación postoperatoria en las unidades de cuidados críticos y un menor riesgo de complicaciones (Tablas 1 y 2). Este aumento en la calidad de atención da lugar a un aumento potencial en la cantidad de atención. El uso eficiente de los recursos permite sin necesidad de cambios en la infraestruc- tura hospitalaria, aumentar la cantidad de prácticas y pacientes atendidos en nuestros centros.

Tabla 1. Regresión lineal múltiple evaluando factores que afectan la duración de la estadía en área de cuidados críticos

\begin{tabular}{|l|c|c|c|}
\hline \multicolumn{1}{|c|}{ Variables } & b & IC $95 \%$ \% & p \\
\hline $\begin{array}{l}\text { Adherencia a } \\
\text { recomendaciones } \\
\text { ERAS (\%) }\end{array}$ & $-0,04$ (noches) & $-0,06$ a $-0,02$ & $<0,0001$ \\
\hline P- Posum (\%) & $+0,09$ (noches) & 0,06 a 0,12 & $<0,0001$ \\
\hline $\begin{array}{l}\text { Abordaje por } \\
\text { laparotomía vs. } \\
\text { Mini invasivo }\end{array}$ & 0,7 a 2 & $<0,0001$ \\
\hline ASA $>3$ & $+0,5$ (noches) & 0,21 a 1 & 0,0001 \\
\hline
\end{tabular}

Solo se presentan aquellas que mostraron una correlación significativa. Una $\mathrm{p}<0,05$ fue considerada significativa. IC: intervalo de confianza; $P$ - Posum: Preoperative risk score $(n=1216)$.

Para que este proyecto llegue a la mayor cantidad posible de pacientes latinoamericanos, es crucial una comunicación efectiva de todos los centros involucrados en la región. Debido a la iniciativa de un grupo de latinoamericanos que asistieron al Congreso Mundial ERAS en 2016, se acordó abrir un capítulo de la Sociedad ERAS en la región de América del Sur y el Caribe. Un año después, ERAS LATAM fue fundado en Uruguay durante el $34^{\circ}$ Congreso de la Confederación Latinoamericana de Sociedades de Anestesiología
Adherencia

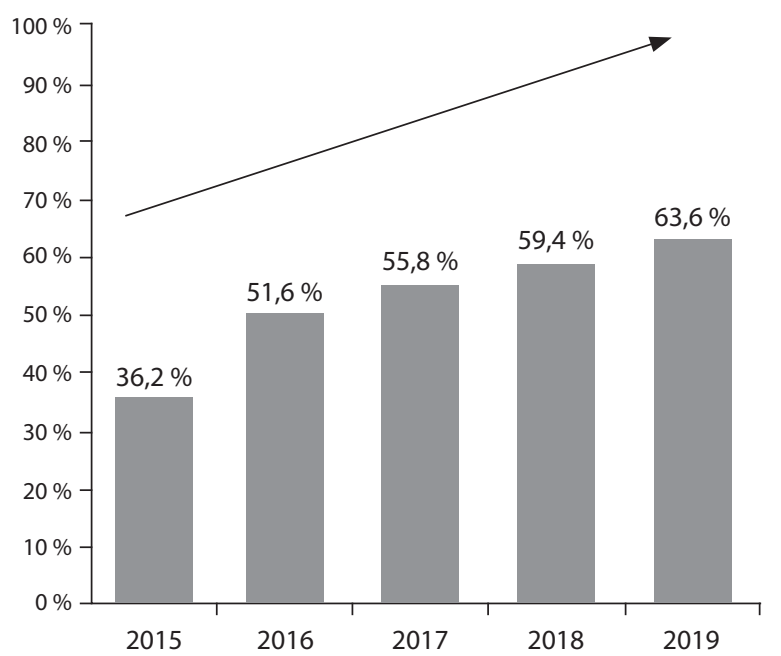

Estadía

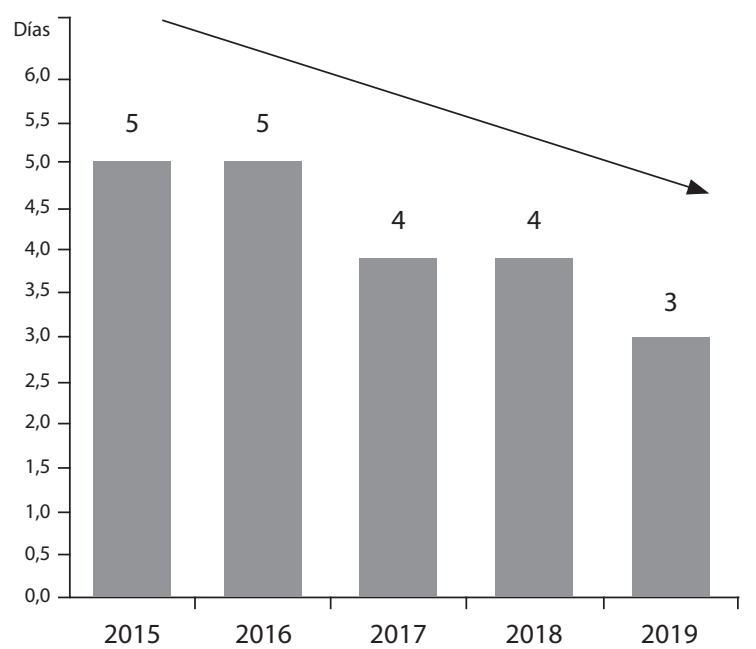

Figura 1. Adherencia y Estadía. Tendencia año a año para toda la región Latinoamericana. 
(CLASA). Desde entonces, se han organizado múltiples foros de intercambio y la promoción de ERAS a lo largo de nuestra región. ERAS LATAM (www.eraslatam.org) constituye la red de colaboración que se necesitaba para brindar orientación y apoyo a las instituciones de nuestra región que buscan iniciar su camino en los programas de recuperación posquirúrgica intensificada. Un gran cambio está al alcance, y nuestro objetivo es hacer que esté disponible para todos.

Tabla 2. Regresión logística evaluando factores de riesgo para complicación postoperatoria

\begin{tabular}{|l|c|c|c|}
\hline \multicolumn{1}{|c|}{ Variables } & OR & IC 95 \% & p \\
\hline $\begin{array}{l}\text { Adherencia a } \\
\text { recomendaciones ERAS (\%) }\end{array}$ & 0,9 & 0,95 a 0,96 & $<0,0001$ \\
\hline P - Posum (\%) & 1,01 & 1,001 a 1,12 & 0,04 \\
\hline $\begin{array}{l}\text { Abordaje por laparotomía vs. } \\
\text { Mini invasivo }\end{array}$ & 1,7 & 1,2 a 2,3 & 0,001 \\
\hline ASA $>3$ & 1,1 & 1,02 a 1,2 & 0,01 \\
\hline
\end{tabular}

Solo se presentan aquellas que mostraron una correlación significativa. Una $\mathrm{p}<0,05$ fue considerada significativa. IC: intervalo de confianza; $\mathrm{P}$ - Posum: Preoperative risk score $(\mathrm{n}=1.216)$.

\section{Financiación}

El presente artículo no tuvo financiación.

\section{Conflicto de intereses}

Los autores declaran no tener conflicto de intereses.

\section{Declaración de autoría}

SML y AAO: Redacción del artículo; OL: Supervisión final. Todos los autores revisaron de artículo y validaron su versión final.

\section{Referencias bibliográficas}

1. Weiser TG, Regenbogen SE, Thompson $\mathrm{KD}$, Haynes $\mathrm{AB}$, Lipsitz SR, Berry WR, et al. An estimation of the global volume of surgery: a modelling strategy based on available data. Lancet. 2008;372(9633):139-44.

2. Weiser TG, Haynes AB, Molina G, Lipsitz SR, Esquivel MM, Uribe-leitz T, et al. Estimate of the global volume of surgery in 2012 : an assessment supporting improved health outcomes. Lancet. 2015;385(Suppl 2):S11.

3. Shrime MG, Bickler SW, Alkire BC, Mock C. Global burden of surgical disease: an estimation from the provider perspective. Lancet Glob Health. 2015;3(Suppl 2):S8-9.

4. Meara JG, Leather AJM, Hagander L, Alkire BC, Alonso N, Ameh EA, et al. Global Surgery 2030 : evidence and solutions for achieving health, welfare, and economic development. Lancet. 2015;386(9993):569-24.

5. Ljunggvist O, Scott M, Fearon KC. Enhanced Recovery After Surgery: A Review. JAMA Surg. 2017;152(3):292-8.

6. Ljunggvist O, Young-Fadok T, Demartines N. The History of Enhanced Recovery After Surgery and the ERAS Society. J Laparoendosc Adv Surg Tech A. 2017;27(9):860-2.

7. Currie A, Soop M, Demartines N, Fearon K, Kennedy R, Ljunggvist O. Enhanced Recovery After Surgery Interactive Audit System: 10 Years' Experience with an International Web-Based Clinical and Research Perioperative Care Database. Clin Colon Rectal Surg. 2019;32(1):75-81.

8. Institute of Medicine of the National Academies. Transforming Clinical Research in the United States: Challenges and Opportunities: Workshop Summary [Internet]. Washington D.C.: The National Academies Press; 2010 [Fecha de consulta: 1 de mayo 2019]. Disponible en: https://www.ncbi.nlm.nih. gov/books/NBK50892/pdf/Bookshelf_NBK50892.pdf

9. Delaney CP, Fazio VW, Senagore AJ, Robinson B, Halverson AL, Remzi FH. 'Fast track' postoperative management protocol for patients with high co-morbidity undergoing complex abdominal and pelvic colorectal surgery. Br J Surg. 2001;88(11):1533-8.

10. Nicholson A, Lowe MC, Parker J, Lewis SR, Alderson P, Smith AF. Systematic review and meta-analysis of enhanced recovery programmes in surgical patients. Br J Surg. 2014; 101 (3): $172-88$. 